\title{
DNA testing and domestic dogs
}

\author{
Cathryn Mellersh
}

Received: 9 August 2011/Accepted: 15 October 2011/Published online: 10 November 2011

(C) The Author(s) 2011. This article is published with open access at Springerlink.com

\begin{abstract}
There are currently about 80 different DNA tests available for mutations that are associated with inherited disease in the domestic dog, and as the tools available with which to dissect the canine genome become increasingly sophisticated, this number can be expected to rise dramatically over the next few years. With unrelenting media pressure focused firmly on the health of the purebred domestic dog, veterinarians and dog breeders are turning increasingly to DNA tests to ensure the health of their dogs. It is ultimately the responsibility of the scientists who identify disease-associated genetic variants to make sensible choices about which discoveries are appropriate to develop into commercially available DNA tests for the lay dog breeder, who needs to balance the need to improve the genetic health of their breed with the need to maintain genetic diversity. This review discusses some of the factors that should be considered along the route from mutation discovery to DNA test and some representative examples of DNA tests currently available.
\end{abstract}

\section{Introduction}

In December 2004 the $\$ 30$ million project funded by the National Human Genome Research Institute (NHGRI) in the United States to sequence the entire dog genome was completed and the results made publicly available. The NHGRI made the decision to fund such an expensive undertaking because it recognised the dog as an unrivalled model organism with which to study the genetics of a wide

C. Mellersh $(\bowtie)$

Canine Genetics, Animal Health Trust, Lanwades Park,

Kentford, Newmarket, Suffolk CB8 7UU, UK

e-mail: cathryn.mellersh@aht.org.uk range of inherited traits, including inherited disease, behaviour, and morphology. Although the NHGRI's motives for sequencing all the DNA in the dog were primarily human-centric, the findings that have emerged from the canine genome project, and that will continue to materialise for many years to come, have profound implications for both veterinary and human medical research. Most importantly, the pace at which genetic variants associated with inherited canine diseases have been identified has increased dramatically and will continue to do so as the tools available to dissect the genetic basis of canine inherited traits become increasingly more sophisticated.

Studies over the last decade, aimed at understanding the genetic basis of inherited traits in the domestic dog, have revealed mutations in dozens of genes, some of them novel, which are now worthy candidates for further study in other species, including man. In addition to mutations associated with Mendelian traits, an ever-increasing number of loci that are associated with genetically complex conditions are being reported. Many of these will almost certainly shed light on the development of similar conditions in other species as well.

A better understanding of the nature and function of some of the genes identified in the dog that are associated with equivalent conditions in man will no doubt lead, in the long term, to improved diagnosis and treatment for human patients. It is also possible that many of these downstream benefits will eventually also be applicable to dogs. But by far the most likely and immediate improvement to the health of domestic dogs will derive from the DNA tests that are routinely made available once a disease-associated mutation has been identified. Currently, DNA tests are available for over 80 different canine mutations, a number that can be expected to increase very quickly in the coming years, and over 120 breeds are able to take advantage of at 
least one DNA test. A list of DNA tests available for dogs at the time of writing is given in Table 1, although it should be noted that new DNA tests are becoming available very rapidly so readers should check with individual testing laboratories for a complete list of tests available. Alternative resources for finding lists of currently available DNA tests are http://www.thekennelclub.org.uk/item/2108, http:// www.offa.org/dna_alltest.html, and http://www.akcchf.org/ canine-health/genetic-tests/. This article does not attempt to discuss every test available. Rather it discusses some of the considerations that should be taken into account to successfully translate scientific findings into robust and useful tools for the lay dog breeder to use, and in so doing it uses a few representative examples of DNA tests that are currently available.

\section{The domestic dog, inherited disease, and DNA testing}

Intense selection, high levels of inbreeding, the extensive use of a limited number of sires, and genetic isolation are all hallmarks of modern breeds of domestic dog. It is widely agreed that part of the collateral damage from these practices is that purebred dogs have a greater risk of suffering from genetically simple inherited disorders than their cross-breed cousins. Intense media interest continues to apply pressure on both dog breeders and the veterinary profession to improve the health of purebred dogs, the result being that these stakeholders are turning increasingly to DNA tests to assist with both breeding decisions and also the diagnostic process. Some dog breeders are exceptionally well informed when it comes to genetics and possess a very good understanding of the inherited disorders that affect their breed(s). But many are not, and they rely on their general practice veterinarian for information, who also may not have a good knowledge of the majority of inherited conditions that affect all the different breeds they encounter.

It is, therefore, ultimately the responsibility of the scientists who identify genetic variants associated with canine inherited disorders to exercise prudence when they make DNA tests available. Purebred dog populations, by definition, are genetically compromised, with many having an unequivocal need to maximise diversity. Breeders are increasingly using DNA tests to shape their breeding decisions, with the need to reduce the frequency of deleterious mutations being balanced with the need to maintain genetic diversity. There is a critical need, therefore, for full and transparent information, written in lay terms, explaining (1) the mode of inheritance and penetrance of specific mutations, (2) the risk of disease associated with specific genotypes, (3) the frequency of each mutation within different breed populations, and (4) the breeds that are genuinely at risk, so that dogs are not unnecessarily eliminated from the breeding population. The existence of phenocopies within the breed, if known, should also be documented.

DNA testing of companion animals is unregulated, and so one of the few ways in which potential "customers" can judge the merit of any DNA test is if a description of the mutation on which the test is based has been accepted by a peer-reviewed scientific publication. However, the data that establish a genetic variant as a provocative candidate for downstream, comparative studies (and therefore worthy of publication) is not always the same information that a dog owner needs to discern whether they should use the DNA test and what decisions to make in light of the results. It is therefore not sufficient for a DNA test provider merely to provide a hyperlink to a peer-reviewed publication without backing it up with some layman-friendly explanation.

It is worth noting that once a mutation has been reported in the scientific literature there is little stopping private, for-profit organisations from offering a genetic test based on a published finding, irrespective of the opinion of the scientists who made the discovery. Therefore, suggesting that researchers have absolute control over which DNA tests are offered might be an oversimplification in some cases. Nevertheless, scientists should certainly be encouraged to offer "user-friendly" advice on how to use genetic tests and to work with dog breed clubs and organisations to disseminate this advice. Perhaps they should also be willing to suggest when consideration of a particular diseaseassociated variant to shape breeding decisions is inappropriate.

\section{DNA tests for recessive mutations}

The vast majority of DNA tests currently available are for autosomal recessive mutations associated with Mendelian or single-gene traits. Some of these traits are morphological, such as coat colour, but most are for disease mutations, many of which are severe and debilitating. Providing advice to breeders for these tests is generally very straightforward; essentially all dogs can be safely bred, regardless of their genotype, provided both the sire and the dam have been tested and carriers and genetically affected dogs are mated only to dogs that are clear of the mutation. Often, the initial reaction of breeders is to avoid breeding with all but homozygous wild-type $(+/+)$ dogs, and indeed many members of the veterinary community who were indoctrinated to never breed affected dogs condone this view. However, if a mutation is frequent within a breed, breeders should be counselled to include carriers in the breeding population, for at least a generation, to avoid 
Table 1 List of DNA tests available for dogs

\begin{tabular}{|c|c|c|}
\hline Breed & Condition / coat colour & Company or testing laboratory \\
\hline \multirow[t]{2}{*}{ Afghan Hound } & Canine Coat and Nose Colour & HealthGene \\
\hline & Canine Mask Test & HealthGene \\
\hline \multirow[t]{2}{*}{ Airedale Terrier } & Haemophilia B & HealthGene, Cornell University \\
\hline & Factor V11 Deficiency & VetGen, Laboklin \\
\hline \multirow[t]{2}{*}{ Alaskan Malamute } & Coat Length & VDA, AHT \\
\hline & Cone Degeneration & Optigen \\
\hline American Eskimo Dog & Progressive Retinal Atrophy, prcd & Optigen \\
\hline American Pit Bull Terrier & $\begin{array}{l}\text { Cone rod dystrophy } 2 \text { (crd2)Cerebellar Ataxia } \\
\text { (NCL-A) }\end{array}$ & Optigen \\
\hline American Staffordshire Terrier & Cerebellar Ataxia (NCL-A) & Optigen \\
\hline \multirow[t]{2}{*}{ Australian Cattle Dog } & Progressive Retinal Atrophy, prcd & OptiGen, Genetic Technologies Ltd, Laboklin \\
\hline & Coat Colour Variations & VetGen \\
\hline \multirow[t]{9}{*}{ Australian Shepherd } & Cone Degeneration (CD) & Optigen \\
\hline & Canine Multi-focal Retinopathy & Optigen \\
\hline & $\begin{array}{l}\text { Collie Eye Anomaly (CEA) / choroidal } \\
\text { hypoplasia }(\mathrm{CH})\end{array}$ & Optigen, Genetic Technologies Ltd \\
\hline & Hereditary Cataract (HC-HSF4) & AHT, Genoscoper \\
\hline & Multi drug resistance (MDR1) & $\begin{array}{l}\text { HealthGene, Genetic Technologies Ltd, } \\
\text { Laboklin, Finnzymes }\end{array}$ \\
\hline & Progressive Retinal Atrophy, prcd & OptiGen, Genetic Technologies Ltd, Laboklin \\
\hline & $\begin{array}{l}\text { Cobalamin Malabsorption (Methylmalonic } \\
\text { Aciduria) }\end{array}$ & PennGen \\
\hline & Canine Coat and Nose Colour & HealthGene \\
\hline & Bobtail gene & AHT, Genoscoper \\
\hline Australian Terrier & von Willebrand's disease & Genetic Technologies Ltd \\
\hline \multirow[t]{2}{*}{ Basenji } & Pyruvate Kinase (PK) Deficiency & $\begin{array}{l}\text { OptiGen, HealthGene, VetGen, PennGen, } \\
\text { Laboklin, Genetic Technologies Ltd, } \\
\text { Genindexe, van Haeringen }\end{array}$ \\
\hline & * Fanconi Syndrome & University of Missouri \\
\hline \multirow[t]{2}{*}{ Basset Hound } & $\begin{array}{l}\text { X-linked severe combined immunodeficiency } \\
\text { (SCID) }\end{array}$ & $\begin{array}{l}\text { PennGen, Laboklin, Genetic Technologies } \\
\text { Ltd }\end{array}$ \\
\hline & Thrombopathia & Auburn University \\
\hline \multirow[t]{3}{*}{ Beagle } & Pyruvate Kinase Deficiency & PennGen, genetic technologies Ltd; Laboklin \\
\hline & Coagulation Factor VII deficiency & AHT, PennGen, VetGen, Laboklin \\
\hline & Musladin-Lueke Syndrome & University of California \\
\hline \multirow[t]{2}{*}{ Bedlington Terrier } & Copper Toxicosis* & $\begin{array}{l}\text { VetGen, HealthGene, AHT, VetGen, van } \\
\text { Haeringen, VDA, Finnzymes, Laboklin, } \\
\text { Genetic Technologies Ltd }\end{array}$ \\
\hline & Copper Toxicosis, COMMD1 & AHT, VetGen, Genetic Technologies Ltd \\
\hline Belgian Shepherd & Canine Coat and Nose Colour & HealthGene \\
\hline - Groenendael & Canine Coat and Nose Colour & HealthGene \\
\hline - Laekenois & Canine Coat and Nose Colour & HealthGene \\
\hline - Malinois & Canine Coat and Nose Colour & HealthGene \\
\hline - Tervueren & Canine Coat and Nose Colour & HealthGene \\
\hline Bernese Mountain Dog & von Willebrand's Disease Type 1 & $\begin{array}{l}\text { VetGen, Finnzymes, Genetic Technologies } \\
\text { Ltd, Laboklin }\end{array}$ \\
\hline
\end{tabular}


Table 1 continued

\begin{tabular}{|c|c|c|}
\hline Breed & Condition / coat colour & Company or testing laboratory \\
\hline \multirow[t]{8}{*}{ Border Collie } & $\begin{array}{l}\text { Collie Eye Anomaly }(\mathrm{CEA}) / \text { choroidal } \\
\text { hypoplasia }(\mathrm{CH})\end{array}$ & Optigen, Genetic Technologies Ltd \\
\hline & Ceroid Lipofuscinosis & $\begin{array}{l}\text { Optigen, Genetic Technologies Ltd, AHT } \\
\text { Laboklin }\end{array}$ \\
\hline & Multi drug resistance (MDR1) & Laboklin, Finnzymes \\
\hline & Canine Cyclic Neutropenia & Laboklin \\
\hline & Canine Coat and Nose Colour & HealthGene \\
\hline & Coat Colour Gene Variations & VetGen \\
\hline & Coat Length & AHT \\
\hline & Trapped Neutrophil Syndrome (TNS) & Laboklin, OptiGen \\
\hline Boston Terrier & Early onset, hereditary cataract (HC-HSF4) & AHT \\
\hline \multirow[t]{3}{*}{ Boxer } & Degenerative Myelopathy & University of Missouri \\
\hline & von Willebrand's Disease Type II & Finnzymes \\
\hline & Bobtail gene & AHT \\
\hline \multirow[t]{2}{*}{ Briard } & Congenital Stationary Night Blindness (CSNB) & $\begin{array}{l}\text { OptiGen, HealthGene, AHT, Laboklin, } \\
\text { Genetic Technologies Ltd, Genindexe, } \\
\text { Antagene, van Haeringen }\end{array}$ \\
\hline & Canine Coat and Nose Colour & Healthgene \\
\hline \multirow[t]{2}{*}{ Brittany } & Canine Coat and Nose Colour & HealthGene \\
\hline & Bobtail gene & Genoscoper \\
\hline Bulldog & Hyperuricosuria & University of CA, AHT \\
\hline \multirow[t]{2}{*}{ Bullmastiff } & Dominant Progressive Retinal Atrophy & Optigen, Laboklin, Genetic Technologies Ltd \\
\hline & Canine Multi-focal Retinopathy & Optigen \\
\hline Bull Terrier & Haemophilia B (Factor IX Deficiency) & HealthGene, Cornell University \\
\hline Bull Terrier (Miniature) & PLL & AHT, OFA \\
\hline \multirow[t]{3}{*}{ Cairn Terrier } & Globoid Cell Leukodystrophy & $\begin{array}{l}\text { HealthGene, Laboklin, Genetic Technologies } \\
\text { Ltd, Genindexe }\end{array}$ \\
\hline & Pyruvate Kinase Deficiency & $\begin{array}{l}\text { PennGen, Genetic Technologies Ltd, } \\
\text { Laboklin }\end{array}$ \\
\hline & Haemophilia B & HealthGene \\
\hline \multirow[t]{2}{*}{ Chihuahua (Long and Smooth) } & Pyruvate Kinase Deficiency & $\begin{array}{l}\text { PennGen, Genetic Technologies Ltd, } \\
\text { Laboklin }\end{array}$ \\
\hline & Coat length & VDA \\
\hline \multirow[t]{2}{*}{ Chinese Crested } & Progressive Retinal Atrophy, pred & OptiGen, Genetic Technologies Ltd, Laboklin \\
\hline & PLL & AHT, OFA \\
\hline \multirow[t]{5}{*}{ Collie ( Rough) } & $\begin{array}{l}\text { Collie Eye Anomaly }(\mathrm{CEA}) / \text { choroidal } \\
\text { hypoplasia }(\mathrm{CH})\end{array}$ & OptiGen, Genetic Technologies Ltd \\
\hline & Multi drug resistance (MDR1) & $\begin{array}{l}\text { HealthGene, Genetic Technologies Ltd, } \\
\text { Laboklin, Finnzymes }\end{array}$ \\
\hline & Progressive Retinal Atrophy, rcd2 & Optigen \\
\hline & Canine Cyclic Neutropenia & HealthGen, Laboklin, Vetgen \\
\hline & Canine Coat and Nose Colour & HealthGene \\
\hline \multirow[t]{5}{*}{ Collie (Smooth) } & $\begin{array}{l}\text { Collie Eye Anomaly (CEA)/choroidal } \\
\text { hypoplasia }(\mathrm{CH})\end{array}$ & OptiGen, Genetic Technologies Ltd \\
\hline & Multi drug resistance (MDR1) & $\begin{array}{l}\text { HealthGene, Genetic Technologies Ltd, } \\
\text { Laboklin, Finnzymes }\end{array}$ \\
\hline & Progressive Retinal Atrophy, rcd2 & Optigen \\
\hline & Canine Cyclic Neutropenia & HealthGene, Laboklin, VetGen \\
\hline & Canine Coat and Nose Colour & HealthGene \\
\hline \multirow[t]{2}{*}{ Coton De Tulear } & Canine Multi-focal Retinopathy & OptiGen \\
\hline & von Willebrand's Disease Type 1 & VetGen \\
\hline
\end{tabular}


Table 1 continued

\begin{tabular}{|c|c|c|}
\hline Breed & Condition / coat colour & Company or testing laboratory \\
\hline \multirow[t]{5}{*}{ Dachshund (Standard Wire Haired) } & Narcolepsy & $\begin{array}{l}\text { OptiGen, HealthGene, Genetic Technologies } \\
\text { Ltd, van Haeringen }\end{array}$ \\
\hline & Pyruvate Kinase Deficiency & $\begin{array}{l}\text { PennGen, Genetic Technologies Ltd, } \\
\text { Laboklin }\end{array}$ \\
\hline & Canine Coat and Nose Colour & HealthGene \\
\hline & Ceroid Lipofuscinosis & University of Missouri \\
\hline & CRD (NPHP4) & AHT \\
\hline Dachshund (Miniature Longhaired) & Progressive Retinal Atrophy, cord-1 & $\begin{array}{l}\text { AHT, Laboklin, University of Missouri, } \\
\text { Genoscoper }\end{array}$ \\
\hline \multirow[t]{2}{*}{ Dachshund (Miniature Smooth Haired) } & Progressive Retinal Atrophy, cord-1 & $\begin{array}{l}\text { AHT, Laboklin, University of Missouri, } \\
\text { Genoscoper }\end{array}$ \\
\hline & Coat length & AHT \\
\hline Dachshund (Miniature Wire Haired) & $\begin{array}{l}\text { Progressive Retinal Atrophy, cord-1 } \\
\text { CRD (NHPH4) }\end{array}$ & AHT \\
\hline \multirow[t]{2}{*}{ Dalmatian } & Hyperuricosuria & AHT \\
\hline & Canine Coat and Nose Colour & HealthGene, VetGen, Laboklin \\
\hline \multirow[t]{2}{*}{ Deerhound } & Factor VII Deficiency & PennGen; VetGen, Laboklin \\
\hline & Coagulation Factor VII deficiency & AHT \\
\hline \multirow[t]{3}{*}{ Dobermann } & Narcolepsy & $\begin{array}{l}\text { OptiGen, HealthGene, Genetic Technologies } \\
\text { Ltd, van Haeringen, Laboklin }\end{array}$ \\
\hline & von Willebrand's Disease Type 1 & $\begin{array}{l}\text { VetGen, Finnzymes, Laboklin, Genetic } \\
\text { Technologies Ltd }\end{array}$ \\
\hline & Canine Coat and Nose Colour & HealthGene \\
\hline Dogue de Bordeaux & Canine Multi-focal Retinopathy & Optigen \\
\hline \multirow[t]{2}{*}{ English Setter } & Ceroid Lipofuscinosis & University of Missouri \\
\hline & Canine Coat and Nose Colour & HealthGene \\
\hline Entlebucher Mountain Dog & Progressive Retinal Atrophy, prcd & OptiGen, Laboklin \\
\hline Finnish Lapphund & Progressive Retinal Atrophy, prcd & OptiGen, Genetic Technologies Ltd, Laboklin \\
\hline \multirow[t]{3}{*}{ French Bulldog } & Hereditary Cataract (HC-HSF4) & AHT \\
\hline & Canine Coat and Nose Colour & HealthGene \\
\hline & Canine Mask Test & HealthGene \\
\hline German Pinscher & von Willebrand's Disease & VetGen, Laboklin \\
\hline German Longhaired Pointer & Canine Coat and Nose Colour & HealthGene \\
\hline \multirow[t]{8}{*}{ German Shepherd Dog } & Mucopolysaccharidosis VII & PennGen, Laboklin \\
\hline & Pyuruvate Kinase Deficiency & Laboklin \\
\hline & Multi drug resistance (MDR1) & Laboklin, Finnzymes \\
\hline & Degenerative Myelopathy & University of Missouri \\
\hline & Dwarfism & Laboklin \\
\hline & Anal Furunculosis & Genoscoper \\
\hline & Canine Coat and Nose Colour & HealthGene \\
\hline & Coat length & VDA, AHT \\
\hline \multirow[t]{3}{*}{ German Shorthaired Pointer } & Cone degeneration & Optigen, Genetic Technologies Ltd \\
\hline & von Willebrand's Disease Type II & VetGen, Finnzymes \\
\hline & Canine Coat and Nose Colour & HealthGene \\
\hline \multirow[t]{3}{*}{ German Wirehaired Pointer } & Haemophilia B & Cornell University \\
\hline & von Willebrand's Disease Type II & $\begin{array}{l}\text { VetGen, University of Utrecht, Laboklin, } \\
\text { Finnzymes }\end{array}$ \\
\hline & Canine Coat and Nose Colour & HealthGene \\
\hline
\end{tabular}


Table 1 continued

\begin{tabular}{|c|c|c|}
\hline Breed & Condition / coat colour & Company or testing laboratory \\
\hline \multirow[t]{2}{*}{ Giant Schnauzer } & $\begin{array}{l}\text { Cobalamin Malabsorption (Methylmalonic } \\
\text { Aciduria) }\end{array}$ & PennGen \\
\hline & Factor VII deficiency & VetGen, Laboklin \\
\hline Glen of Imaal Terrier & Progressive Retinal Atrophy, crd-3 & Optigen, Bochum University \\
\hline \multirow[t]{2}{*}{ Gordon Setter } & Progressive Retinal Atrophy, rcd-4 & AHT \\
\hline & Coat Colour Gene Variations & VetGen \\
\hline \multirow[t]{2}{*}{ Great Dane } & Canine Coat and Nose Colour & HealthGene \\
\hline & Canine Mask Test & HealthGene \\
\hline \multirow[t]{3}{*}{ Greyhound } & Polyneuropathy/Neuropathy (NDRG1) & Optigen \\
\hline & Canine Coat and Nose Colour & HealthGene \\
\hline & Canine Mask Test & HealthGene \\
\hline Hungarian Kuvasz & Progressive Retinal Atrophy, pred & Genetic Technologies, OptiGen \\
\hline \multirow[t]{3}{*}{ Irish Red \& White Setter } & $\begin{array}{l}\text { Canine Leucocyte Adhesion Deficiency } \\
\text { (CLAD) }\end{array}$ & OptiGen, AHT, Genindexe \\
\hline & Progressive Retinal Atrophy, rcd-1 & Optigen \\
\hline & von Willebrand's disease & AHT \\
\hline \multirow[t]{3}{*}{ Irish Setter } & $\begin{array}{l}\text { Canine Leucocyte Adhesion Deficiency } \\
\text { (CLAD) }\end{array}$ & $\begin{array}{l}\text { OptiGen, VetGen, HealthGene, AHT, } \\
\text { Laboklin, Genetic Technologies Ltd, } \\
\text { Genindexe, van Haeringen }\end{array}$ \\
\hline & Progressive Retinal Atrophy, rcd-1 & $\begin{array}{l}\text { OptiGen, VetGen, HealthGene, AHT, } \\
\text { Laboklin, Genetic Technologies Ltd, } \\
\text { Genindexe, Antagene }\end{array}$ \\
\hline & Progressive Retinal Atrophy, rcd-4 & AHT \\
\hline Italian Spinone & Cerebellar Ataxia & AHT \\
\hline Japanese Chin & Canine Coat and Nose Colour & HealthGene \\
\hline Japanese Shiba Inu & Coat Length & VDA \\
\hline Keeshond & PHPT & Cornell University \\
\hline \multirow[t]{2}{*}{ Kerry Blue Terrier } & von Willebrand's Disease Type I & $\begin{array}{l}\text { VetGen, Finnzymes, Genetic Technologies } \\
\text { Ltd, PennGen }\end{array}$ \\
\hline & Factor XI Deficiency & PennGen \\
\hline Kooikerhondje & von Willebrand's Disease Type II & Finnzymes, VetGen, University of Utrecht \\
\hline Lagotto Romagnolo & Juvenile epilepsy & Genoscoper \\
\hline \multirow[t]{2}{*}{ Lancashire Heeler } & $\begin{array}{l}\text { Collie Eye Anomaly (CEA)/choroidal } \\
\text { hypoplasia }(\mathrm{CH})\end{array}$ & Optigen \\
\hline & PLL & AHT, OFA \\
\hline \multirow[t]{3}{*}{ Large Munsterlander } & Hyperuricosuria & AHT \\
\hline & Canine Coat and Nose Colour & HealthGene \\
\hline & Black Hair Follicular Dysplasia & HealthGene \\
\hline \multirow[t]{2}{*}{ Lhasa Apso } & Renal Dysplasia* & VetGen, Genetic Technologies Ltd \\
\hline & Haemophilia B & HealthGene, Cornell University \\
\hline Lowchen & Canine Coat and Nose Colour & HealthGene \\
\hline Manchester Terrier & von Willebrand's Disease, Type 1 & $\begin{array}{l}\text { VetGen, Finnzymes, Laboklin, Genetic } \\
\text { Technologies Ltd }\end{array}$ \\
\hline \multirow[t]{2}{*}{ Mastiff } & Dominant Progressive Retinal Atrophy & $\begin{array}{l}\text { OptiGen, Laboklin, Genetic Technologies } \\
\text { Ltd, Genindexe, Antagene }\end{array}$ \\
\hline & Canine Multi-focal Retinopathy & Optigen \\
\hline \multirow[t]{3}{*}{ Miniature Schnauzer } & Progressive Retinal Atrophy, Type A & $\begin{array}{l}\text { OptiGen, Genetic Technologies Ltd, } \\
\text { Genindexe }\end{array}$ \\
\hline & Myotonia congenital & $\begin{array}{l}\text { HealthGene, PennGen, OptiGen, Laboklin, } \\
\text { Genetic Technologies Ltd }\end{array}$ \\
\hline & Mucopolysaccharidosis & PennGen \\
\hline
\end{tabular}


Table 1 continued

\begin{tabular}{|c|c|c|}
\hline Breed & Condition / coat colour & Company or testing laboratory \\
\hline Miniature Pinscher & Mucopolysaccharidosis VI & PennGen, Genetic Technologies Ltd \\
\hline \multirow[t]{3}{*}{ Newfoundland } & Cystinuria & $\begin{array}{l}\text { OptiGen, HealthGene, PennGen, VetGen, van } \\
\text { Haeringen, VDA, Laboklin, Genetic } \\
\text { Technologies Ltd }\end{array}$ \\
\hline & Canine Coat and Nose Colour & HealthGene \\
\hline & Coat Colour Gene Variations & VetGen \\
\hline Norwegian Elkhound & Progressive Retinal Atrophy, prcd & Optigen \\
\hline Old English Sheepdog & Multi drug resistance (MDR1) & $\begin{array}{l}\text { HealthGene, Genetic Technologies Ltd, } \\
\text { Laboklin, Finnzymes }\end{array}$ \\
\hline Otterhound & Glanzmann's Thrombasthenia Type 1 & Auburn University \\
\hline \multirow[t]{2}{*}{ Papillion } & Von Willebrand's Disease Type 1 & $\begin{array}{l}\text { VetGen, Finnzymes, Genetic Technologies } \\
\text { Ltd }\end{array}$ \\
\hline & PRA & Genoscoper \\
\hline Parson Russell Terrier & PLL & AHT, OFA \\
\hline \multirow[t]{2}{*}{ Pointer } & Canine Coat and Nose Colour & HealthGene \\
\hline & Coat Colour Gene Variation & VetGen \\
\hline Polish Lowland Sheepdog & Bobtail gene & Genoscoper \\
\hline Pomeranian & Canine Coat and Nose Colour & HealthGene \\
\hline \multirow[t]{3}{*}{ Poodle (Miniature) } & Progressive Retinal Atrophy, prcd & OptiGen, Genetic Technologies Ltd, Laboklin \\
\hline & von Willebrand's Disease Type 1 & $\begin{array}{l}\text { VetGen, Finnzymes, Laboklin, Genetic } \\
\text { Technologies Ltd }\end{array}$ \\
\hline & Canine Coat and Nose Colour & HealthGene, VetGen \\
\hline \multirow[t]{5}{*}{ Poodle (Standard) } & Neonatal Encephalopathy & University of Missouri, VetGen \\
\hline & von Willebrand's Disease Type 1 & $\begin{array}{l}\text { VetGen, Finnzymes, Laboklin, Genetic } \\
\text { Technologies Ltd }\end{array}$ \\
\hline & Degenerative Myelopathy & University of Missouri \\
\hline & Neonatal Encephalopathy with Seizures & University of Missouri \\
\hline & Canine Coat and Nose Colour & HealthGene, VetGen \\
\hline \multirow[t]{3}{*}{ Poodle (Toy) } & Progressive Retinal Atrophy, pred & OptiGen, Genetic Technologies Ltd, Laboklin \\
\hline & von Willebrand's Disease Type 1 & $\begin{array}{l}\text { VetGen, Finnzymes, Laboklin, Genetic } \\
\text { Technologies Ltd }\end{array}$ \\
\hline & Canine Coat and Nose Colour & HealthGene, VetGen \\
\hline \multirow[t]{4}{*}{ Portuguese Water Dog } & Improper Coat (IC13) & Optigen \\
\hline & Progressive Retinal Atrophy, prcd & OptiGen, Genetic Technologies Ltd, Laboklin \\
\hline & GM1 gangliosidosis & HealthGene, Genetic Technologies Ltd \\
\hline & Canine Coat and Nose Colour & HealthGene \\
\hline Pug & Canine Coat and Nose Colour & HealthGene \\
\hline \multirow[t]{2}{*}{ Pyrenean Mountain Dog (Great Pyrenees) } & Glanzmann's Thrombasthenia Type 1 & Auburn University \\
\hline & Canine Multi-focal Retinopathy & OptiGen \\
\hline \multirow[t]{3}{*}{ Retriever (Chesapeake Bay) } & Progressive Retinal Atrophy, pred & OptiGen, Laboklin \\
\hline & Degenerative Myelopathy & University of Missouri \\
\hline & Exercise Induced Collapse & Laboklin \\
\hline \multirow[t]{3}{*}{ Retriever (Curly Coated) } & Glycogenosis (GSD) Type IIIa & Michigan State \\
\hline & Exercise Induced Collapse & Laboklin \\
\hline & Canine Coat and Nose Colour & HealthGene \\
\hline \multirow[t]{2}{*}{ Retriever (Flat Coated) } & Canine Coat and Nose Colour & HealthGene, VetGen, Laboklin \\
\hline & Yellow coat & AHT, Finnzymes, Laboklin \\
\hline \multirow[t]{3}{*}{ Retriever (Golden) } & Muscular Dystrophy & HealthGene, Laboklin \\
\hline & Progressive Retinal Atrophy, prcd & Optigen, Laboklin \\
\hline & Progressive Retinal Atrophy (GR PRA1) & AHT \\
\hline
\end{tabular}


Table 1 continued

\begin{tabular}{|c|c|c|}
\hline Breed & Condition / coat colour & Company or testing laboratory \\
\hline \multirow[t]{10}{*}{ Retriever (Labrador) } & Cystinuria & PennGen, Genetic Technologies Ltd \\
\hline & Narcolepsy & $\begin{array}{l}\text { OptiGen, HealthGene, Genetic Technologies } \\
\text { Ltd, van Haeringen, Laboklin }\end{array}$ \\
\hline & Progressive Retinal Atrophy, prcd & OptiGen, Genetic Technologies Ltd, Laboklin \\
\hline & Retinal Dyslasia/OSD & Optigen \\
\hline & Haemophilia B & HealthGene, Cornell University \\
\hline & Labrador Myopathy (CNM) & AHT, Alfort School, Laboklin, VetGen \\
\hline & Exercise Induced Collapse & Laboklin \\
\hline & Yellow coat & AHT, Laboklin \\
\hline & Canine Coat and Nose Colour & HealthGene, VetGen, Laboklin, Genindexe \\
\hline & Coat Colour Gene Variations & VetGen, Finnzymes \\
\hline \multirow[t]{2}{*}{ Retriever (Nova Scotia Duck Tolling) } & Progressive Retinal Atrophy, pred & OptiGen, Genetic Technologies Ltd, Laboklin \\
\hline & $\begin{array}{l}\text { Collie Eye Anomaly }(\mathrm{CEA}) / \text { choroidal } \\
\text { hypoplasia }(\mathrm{CH})\end{array}$ & OptiGen, Genetic Technologies Ltd \\
\hline Rhodesian Ridgeback & Degenerative Myelopathy & University of Missouri \\
\hline Rottweiler & Coat Length & VDA \\
\hline Russian Black Terrier & Hyperuricosuria & University of CA, AHT \\
\hline \multirow[t]{3}{*}{ Samoyed } & Progressive Retinal Atrophy, X-linked & $\begin{array}{l}\text { OptiGen, Genetic Technologies Ltd, } \\
\text { Antagene }\end{array}$ \\
\hline & Retinal Dyslasia/OSD & Optigen \\
\hline & Hereditary Nephritis & VetGen \\
\hline \multirow[t]{3}{*}{ Schipperke } & Mucopolysaccharidosis IIIB & PennGen \\
\hline & Coat Colour Gene Variation & VetGen \\
\hline & Bobtail gene & Genoscoper \\
\hline \multirow[t]{2}{*}{ Scottish Terrier } & von Willebrand's Disease Type III & VetGen, Laboklin, Genetic Technologies Ltd \\
\hline & Coat Colour Gene Variation & VetGen \\
\hline Sealyham Terrier & PLL & AHT, OFA \\
\hline Shar Pei & Canine Coat and Nose Colour & HealthGene \\
\hline \multirow[t]{4}{*}{ Shetland Sheepdog } & von Willebrand's Disease Type III & VetGen, Laboklin, Genetic Technologies Ltd \\
\hline & $\begin{array}{l}\text { Collie Eye Anomaly (CEA)/choroidal } \\
\text { hypoplasia }(\mathrm{CH})\end{array}$ & Optigen, Genetic Technologies Ltd \\
\hline & Multi drug resistance (MDR1) & $\begin{array}{l}\text { HealthGene, Genetic Technologies Ltd, } \\
\text { Laboklin, Finnzymes }\end{array}$ \\
\hline & Canine Coat and Nose Colour & HealthGene \\
\hline Shih Tzu & Renal Dysplasia* & VetGen, Genetic Technologies Ltd \\
\hline \multirow[t]{2}{*}{ Siberian Husky } & Progressive Retinal Atrophy, X-linked & $\begin{array}{l}\text { OptiGen, Genetic Technologies Ltd, } \\
\text { Anatagene }\end{array}$ \\
\hline & GM1-gangliosidosis & Laboklin \\
\hline Silky Terrier & Progressive Retinal Atrophy, prcd & Optigen \\
\hline Sloughi & Progressive Retinal Atrophy, rcd-1a & $\begin{array}{l}\text { OptiGen, HealthGene, AHT, Laboklin, } \\
\text { Genindexe, Antagene, Genetic Technologies } \\
\text { Ltd }\end{array}$ \\
\hline Soft Coated Wheaten Terrier & Renal Dysplasia* & VetGen, Genetic Technologies Ltd \\
\hline \multirow[t]{3}{*}{ Spaniel (American Cocker) } & Progressive Retinal Atrophy, prcd & $\begin{array}{l}\text { OptiGen, Genindexe, Genetic Technologies } \\
\text { Ltd, Laboklin }\end{array}$ \\
\hline & Phosphofructokinase (PFK) Deficiency & $\begin{array}{l}\text { OptiGen, Health Gene, VetGen, PennGen, } \\
\text { AHT, Genetic Technologies Ltd, } \\
\text { Genindexe, Laboklin }\end{array}$ \\
\hline & Coat Colour Variations & VetGen \\
\hline
\end{tabular}


Table 1 continued

\begin{tabular}{|c|c|c|}
\hline Breed & Condition / coat colour & Company or testing laboratory \\
\hline Spaniel (Clumber) & $\begin{array}{l}\text { Pyruvate Dehydrogenase Phosphatase } \\
\text { Deficiency (PDP 1) }\end{array}$ & $\begin{array}{l}\text { Genetic Technologies Ltd, University of } \\
\text { Missouri, AHT, Laboklin; Genoscoper }\end{array}$ \\
\hline \multirow[t]{4}{*}{ Spaniel (Cocker) } & Progressive Retinal Atrophy, prcd & OptiGen, Genetic Technologies Ltd, Laboklin \\
\hline & Phosphofructokinase (PFK) Deficiency & $\begin{array}{l}\text { OptiGen, VDA, HealthGene, van Haeringen, } \\
\text { Genindexe }\end{array}$ \\
\hline & Familial Nephropathy & Optigen, Genetic Technologies Ltd, Anatgene \\
\hline & Canine Coat and Nose Colour & HealthGene \\
\hline \multirow[t]{4}{*}{ Spaniel (English Springer) } & Fucosidosis & $\begin{array}{l}\text { AHT, PennGen, Finnzymes, Laboklin, } \\
\text { Genetic Technologies Ltd, Genindexe, } \\
\text { Finnzymes }\end{array}$ \\
\hline & Phosphofructokinase (PFK) Deficiency & $\begin{array}{l}\text { OptiGen, VetGen, HealthGene, AHT, } \\
\text { PennGen, VDA, Laboklin, Genetic } \\
\text { Technologies Ltd, Genindexe, van } \\
\text { Haeringen, University of Missouri }\end{array}$ \\
\hline & PRA - cord 1 & $\begin{array}{l}\text { AHT, Finnzymes, Laboklin, University of } \\
\text { Missouri, Genoscoper }\end{array}$ \\
\hline & Canine Coat and Nose Colour & HealthGene \\
\hline Spaniel (Field) & Canine Coat and Nose Colour & HealthGene \\
\hline Spaniel (Sussex) & $\begin{array}{l}\text { Pyruvate Dehydrogenase Phosphatase } \\
\text { Deficiency (PDP 1) }\end{array}$ & $\begin{array}{l}\text { AHT, Genetic Technologies Ltd, University } \\
\text { of Missouri, Laboklin, Genoscoper }\end{array}$ \\
\hline \multirow[t]{2}{*}{ Spanish Water Dog } & Progressive Retinal Atrophy, pred & OptiGen, Genetic Technologies Ltd \\
\hline & Bobtail gene & Genoscoper \\
\hline \multirow[t]{3}{*}{ Staffordshire Bull Terrier } & Hydroxyglutaric acidurea, L-2-HGA & AHT, University of Missouri, Laboklin \\
\hline & Hereditary cataract , HC-1 & AHT \\
\hline & Canine Coat and Nose Colour & HealthGene \\
\hline Swedish Lapphund & Progressive Retinal Atrophy, prcd & OptiGen, Genetic Technologies Ltd, Laboklin \\
\hline Swedish Vallhund & Bobtail gene & Genoscoper \\
\hline \multirow[t]{2}{*}{ Tibetan Terrier } & PLL & AHT, OFA, University of Missouri \\
\hline & NCL & OFA \\
\hline Weimaraner & Coat Length & AHT, VDA \\
\hline \multirow[t]{5}{*}{ Welsh Corgi (Cardigan) } & Progressive Retinal Atrophy, rcd-3 & $\begin{array}{l}\text { OptiGen, HealthGene, Laboklin, Genetic } \\
\text { Technologies Ltd, Genindexe, Anatagene, } \\
\text { VetGen, University of Michigan }\end{array}$ \\
\hline & $\begin{array}{l}\text { X-linked severe combined immunodeficiency } \\
\text { (SCID) }\end{array}$ & $\begin{array}{l}\text { PennGen, Laboklin, Genetic Technologies } \\
\text { Ltd }\end{array}$ \\
\hline & Degenerative Myelopathy & University of Missouri \\
\hline & Canine Coat and Nose Colour & HealthGene \\
\hline & Coat Length & VDA \\
\hline \multirow[t]{5}{*}{ Welsh Corgi (Pembroke) } & von Willebrand's Disease TypeI & $\begin{array}{l}\text { VetGen, Finnzymes, Genetic Technologies } \\
\text { Ltd, Laboklin }\end{array}$ \\
\hline & Severe combined immunodeficiency (SCID) & PennGen, Genetic Technologies Ltd \\
\hline & Degenerative Myelopathy & University of Missouri \\
\hline & Coat length & AHT, VDA \\
\hline & Bobtail gene & AHT \\
\hline \multirow[t]{2}{*}{ West Highland White Terrier } & Pyruvate Kinase (PK) Deficiency & $\begin{array}{l}\text { HealthGene, PennGen, VDA, Laboklin, } \\
\text { Genetic Technologies Ltd, Genindexe, } \\
\text { VetGen }\end{array}$ \\
\hline & Globoid Cell Leukodystrophy & $\begin{array}{l}\text { HealthGene, Laboklin, Genetic Technologies } \\
\text { Ltd, Genindexe }\end{array}$ \\
\hline
\end{tabular}


Table 1 continued

\begin{tabular}{lll}
\hline Breed & Condition / coat colour & Company or testing laboratory \\
\hline Whippet & Collie Eye Anomaly (CEA)/choroidal & Optigen \\
& hypoplasia (CH) - Longhaired Whippets only & \\
& Canine Multidrug Sensitivity Test (MDRI) & HeathGene \\
& Canine Coat and Nose Colour & HealthGene \\
& Canine Mask Test & HealthGene \\
Yorkshire Terrier & Progressive Retinal Atrophy, prcd & Optigen \\
\hline
\end{tabular}

${ }^{a}$ Linked marker test, not a mutation-based, gene test

reducing diversity unnecessarily. Recent data from the 1,000 genomes project revealed that humans carry, on average, between 250 and 300 recessive mutations and at least 50 mutations previously associated with inherited disorders, and it seems reasonable to assume the average dog will carry the same burden of disease-associated variants (Durbin et al. 2010). Expecting breeding dogs to be clear of all risk alleles, therefore, is unrealistic and will severely jeopardise breed diversity.

Recessive mutations for late-onset conditions are notoriously difficult to eliminate if a DNA test is not available, and, in the absence of effective selective pressure, can become common within a breed. Nevertheless, robust data regarding mutation frequency are often unavailable, possibly because the data are rarely required for publication, and as the number of DNA tests available increases, it will become increasingly important for veterinarians and breeders to be able to sensibly prioritise tests for specific breeds. DNA test providers should be prepared to provide accurate information regarding the frequency of a specific mutation in relevant breeds, both when a DNA test is initially made available and at regular intervals, so progress toward the genetic improvement of the breed can be monitored. Obtaining such estimates might require sampling of additional individual dogs from a cross section of the gene pool because DNA samples collected during a genetic investigation are usually biased toward affected individuals and closely related dogs.

Numerous DNA tests are available for autosomal recessive mutations, far too many to describe individually, so this review summarises two representative examples, along with background behind their identification. Both mutations have been reported in the scientific literature and both are relevant to a large number of breeds. The mutations are those for progressive rod cone degeneration (prcd) (Zangerl et al. 2006) and primary lens luxation (PLL) (Farias et al. 2010).

Progressive rod cone degeneration (prcd) is a late-onset form of progressive retinal atrophy (PRA) that affects multiple breeds. Prior to characterization of this disease at the molecular level, elegant interbreed crosses were undertaken to determine that the phenotypically similar diseases that were segregating in multiple breeds, including the miniature poodle, the English and American cocker spaniels, the Labrador retriever, the Australian cattle dog, the Nova Scotia duck tolling retriever, and the Portuguese water dog, were in fact allelic (Aguirre and Acland 1988, 2006). However, when prcd-affected dogs were mated to PRA-affected dogs of the Border Collie, Basenji, and Italian greyhound breeds, the progeny were normal, indicating that these breeds are affected by genetically distinct forms of disease. The pred locus was mapped to a large region on CFA9 in 1998 (Acland et al. 1998), before the canine genome sequence was available and while tools with which to investigate the canine genome were relatively unsophisticated compared to those available today. The whole-genome radiation panels that were available at the time, and that would have been useful to investigate any other region of the genome, did not significantly help to locate the mutation because they were both TK1 selected (Priat et al. 1998), and since TK1 was tightly linked to the prcd locus, it was difficult to order positional candidate genes within the pred critical region. However, the fact that a genetically identical disease segregated in so many breeds proved to be invaluable as it allowed the use of linkage equilibrium mapping across affected breeds to considerably narrow the prcdassociated region (Goldstein et al. 2006) and led to the eventual identification of a single nucleotide substitution in the second codon of a previously unknown gene that is now known to be the cause of pred in over 30 different breeds (Zangerl et al. 2006). A DNA test for the pred mutation is provided by OptiGen (www.optigen.com), a service company cofounded by ophthalmologists Gregory Acland and Gustavo Aguirre to provide DNA-based diagnoses and information about inherited diseases of dogs. The company provides breeding advice as well as comprehensive, breed-specific information for owners 
regarding, for example, the average age of onset of prcd in specific breeds and whether genetically distinct forms of PRA are known to exist in the same breed.

The mutation for PLL is another example of a mutation that segregates in multiple breeds, all of which can take advantage of a DNA test. PLL is an inherited deficiency of the lens suspensory apparatus, the zonule, which is a system of fibres that suspend the lens from the ciliary body, maintaining it on the visual axis and in contact with the anterior surface of the vitreous body. In dogs affected with PLL, ultrastructural abnormalities of the zonular fibres are already evident at 20 months of age (Curtis 1983), long before the lens luxation that typically occurs when the dogs are 3-8 years old, as a result of degeneration and breakdown of the zonules that cause the lens to be displaced from its normal position within the eye (Curtis 1990; Curtis and Barnett 1980; Curtis et al. 1983; Morris and Dubielzig 2005). In the majority of cases the dislocated lens will pass into the anterior chamber where its presence is likely to cause acute glaucoma. The condition has been recognized as a canine familial disorder for more than 100 years (Gray 1909, 1932) and is encountered at high frequency in several terrier breeds and in some other breeds with probable terrier coancestry (Curtis 1990; Curtis and Barnett 1980; Curtis et al. 1983; Morris and Dubielzig 2005; Willis et al. 1979). In 2010 a mutation in ADAMTS17 was described as the cause of PLL in three breeds, the Miniature Bull terrier, the Lancashire Heeler, and the Jack Russell terrier. The mutation is a $\mathrm{G} \rightarrow \mathrm{A}$ substitution at c. $1473+1$, which destroys a splice donor recognition site in intron 10 and causes exon skipping that results in a frameshift and the introduction of a premature termination codon (Farias et al. 2010). A subsequent publication, targeted at a veterinarian audience, reports 14 additional breeds in which the identical mutation segregates, and documents the frequency of the mutation in a subset of breeds (Gould et al. 2011). The great majority of PLL-affected dogs are homozygous for the mutation, but a small minority are heterozygous, leading to speculation that carriers, of some breeds at least, might be at increased risk of developing the condition compared to dogs that are homozygous for the wild-type allele (Farias et al. 2010). A DNA test for the ADAMTS17 substitution is available via each of the two research groups that collaborated to identify the mutation, the Animal Health Trust (www.aht.org.uk) and the University of Missouri, via The Orthopaedic Foundation for Animals (http://www.offa.org/dnatesting/pll.html), as well as a number of other DNA-testing providers that have used the published data to develop their own tests. Both the Animal Health Trust and The Orthopaedic Foundation for Animals provide extensive information regarding the clinical aspects of PLL, the risk to dogs with each of the three possible genotypes of developing PLL, and also breeding advice for owners. The mutation is frequent in several breeds, so advice is provided that counsels breeders to include heterozygotes in the breeding population to avoid pushing breeds through a genetic bottleneck. Both prcd and PLL are examples of where rigorous scientific investigations have provided the data for DNA tests that are provided in a suitable context for dog owners and breeders to use to full advantage.

Validating which breeds a specific DNA test should be usefully offered to is an important consideration for the DNA test provider, and many DNA test providers, including the Animal Health Trust and OptiGen (N. Holmes and S. Pearce-Kelling, personal communications), have a policy of restricting all tests to only those breeds in which the mutation has previously been identified and is also associated with disease. The risk associated with specific mutations might vary, depending on the genetic background, so simply establishing that a mutation is segregating within a breed does not necessarily justify making the test available to that breed.

Both pred and PLL are genetically "simple" conditions, that is, the mutations are completely penetrant and homozygous dogs invariably develop the associated condition during their lives. Examples are emerging of recessive conditions for which the associated mutation is not completely penetrant, indicating that other factors, either genetic or environmental, play a role in the development of the disease. Researchers need to provide dog breeders with specific counselling for such conditions to ensure that they appreciate that not all dogs that are homozygous for disease-associated variants will develop clinical signs during their lives, but that they will pass these mutations onto their offspring (who may inherit different modifier alleles and be affected).

One example of a disease that segregated as a Mendelian trait within an inbred research colony but appeared genetically more complex in the general pet population is a form of retinal degeneration described in the miniature long-haired dachshund (MLHD). The disease was originally described as an early-onset, autosomal recessive PRA, with all affected dogs within an inbred research colony displaying ophthalmologic abnormalities that were detectable by electroretinogram (ERG) by 6 weeks of age and by fundoscopy at 25 weeks of age. The dogs were invariably blind by the time they were 2 years of age (Curtis and Barnett 1993). A subsequent ERG study identified an initial reduction of the cone photoreceptor function which led to the condition being reclassified as a cone-rod dystrophy (CRD) rather than a rod-led PRA, and the disease was termed cord 1 for cone-rod degeneration 1 (Turney et al. 2007). The same condition has also been referred to by others as crd 4 for cone-rod degeneration 4 
(Aguirre and Acland 2006). Using the same colony of dogs, cord1 was mapped to a large region on CFA15, and a mutation in RPGRIPl was identified that cosegregated completely with cord1 in the research colony (Mellersh et al. 2006a). The mutation is a 44-bp insertion of an A29 tract flanked by a 15-bp duplication in exon 2 of the gene, which creates a frameshift and introduces a premature stop codon early in exon 3. Mutations in RPGRIPI have been associated with Leber congenital amaurosis (LCA) (Dryja et al. 2001), retinitis pigmentosa (RP) (Booij et al. 2005), and CRD (Hameed et al. 2003) in humans, as well as inherited retinal abnormalities in mice (Zhao et al. 2003), which suggests that it plays an important role in visual function. Within the research colony of MLHDs there was complete correlation between the RPGRIP1 genotype and phenotype of the dogs with respect to their cord1 phenotype, whereas in the pet MLHD population this was not the case (Miyadera et al. 2009). Outside of the colony there was considerable variation in the age of onset of retinal degeneration in dogs that were homozygous for the RPGRIPI insertion (RPGRIP1 $\left.{ }^{-/}\right)$, which has also been identified in other breeds, including the English Springer Spaniel (ESS) and the Beagle. However, all RPGRIP1 ${ }^{-/-}$ Beagles and MLHDs showed reduced or absent ERG cone responses, even in the absence of ophthalmoscopic abnormalities, a finding that has also been corroborated by Busse et al. (2011). Together, these findings suggest that additional mutations which modify the age of onset of ophthalmoscopic abnormalities associated with the RPGRIP1 mutation are involved. Because the original research colony used was developed from a very small number of dogs, it is likely that the colony was fixed for these additional loci which, therefore, went undetected until the more outbred pet population was investigated. A recent association study using $R P G R I P 1^{-/-}$MLHDs that had either early- or late-onset cord1 has indeed revealed a second locus that segregates with early-onset disease (K. Miyadera, Cambridge, 2010, personal communication), indicating that early-onset CRD in MLHDs is more likely to be a digenic condition and that the RPGRIPI insertion alone causes a late-onset CRD, although ERG abnormalities may be detected early in life. The RPGRIPI insertion is very frequent within both MLHD and ESS populations, probably due to the lack of effective selection against the late-onset CRD with which it is associated. Breeders should thus be mindful to breed with carriers so that the genetic diversity of their breed(s) is not unduly compromised and to consider the RPGRIP1 genotype of their dogs alongside all the other factors they use to weigh a dog's breeding potential.

Two additional examples of recessive mutations that are not completely penetrant but are highly associated with disease (and therefore sound candidates for a DNA test) are those for degenerative myelopathy (DM) in the Boxer, Cardigan Welsh Corgi, Chesapeake Bay Retriever, German Shepherd, Kerry Blue Terrier, Pembroke Welsh Corgi, Rhodesian Ridgeback, and Standard Poodle and hyperuricosuria.

DM is a severe, incurable disease of the spinal cord where demyelination and axonal loss contribute to degeneration of the white matter of the spinal cord resulting in progressive paralysis. The disease has an insidious onset, typically between 8 and 14 years of age, so affected dogs may well have been bred well before their clinical signs develop. The mutation that is tested for, a single-nucleotide missense mutation in SOD1, greatly increases an individual dog's risk of developing DM, although a minority of dogs that carry two copies of the mutation remain free of clinical signs associated with the condition during their lifetime. It is suspected that there are additional mutations and/or environmental factors that modify the effects of the DM mutation and explain why some dogs remain healthy (Awano et al. 2009). The orthopaedic foundation for animals (OFA) makes it very clear on their DNA-testing website that the DM carrier status of dogs should be considered alongside other factors, such as breed type and temperament, and that breeders should not "over-emphasize DNA test results" (http://www.offa.org/dnatesting/ dmbreederguide.html).

Another example of a disease-associated risk factor that is incompletely penetrant and for which there is a commercially available DNA test is the mutation associated with hyperuricosuria, or elevated levels of uric acid in the urine. This trait predisposes dogs to form stones in their bladders, or sometimes kidneys, which often have to be removed surgically and can be difficult to treat. The associated variant, a missense mutation in SLC2A9, is associated with hyperuricosuria in several different breeds, including the Dalmatian (a breed that is fixed for the mutation), but as with DM, not all dogs that are homozygous for the mutation develop urate stones, indicating that other factors are involved (Bannasch et al. 2008; Karmi et al. 2010). The DNA tests for these and other diseaseassociated variants provide dog breeders with invaluable tools with which to reduce the frequency of inherited diseases from breeds at risk, and, provided the mutation being tested for is highly associated with the disease in question, it is difficult to justify not making such DNA tests available to the public. Provided full information is available to enable the nonscientist to understand the level of risk involved, that the mutation is not fully penetrant and that other factors might contribute to the development of disease, then breeds will certainly benefit in the long term from the availability of such tests. 


\section{DNA tests for dominant mutations}

Dominant mutations obviously present dog breeders with a different dilemma from recessive mutations; because all offspring that inherit a disease-associated dominant mutation will develop clinical signs at some stage during their lives, it is harder to justify breeding with animals that carry dominant mutations. Depending on the frequency of the mutation within a population, tests for dominant mutations have the potential to result in more dogs being prevented from breeding, and consequently do more damage to the genetic diversity of a breed, than DNA tests for recessive mutations. As a result, researchers have an even greater responsibility to limit the availability of DNA tests for dominant mutations to those that are highly associated with disease. They need to exercise sensible restraint for mutations that have incomplete penetrance (particularly if the level of penetrance is very low), especially if the disease-associated mutation is frequent within a breed. There are several DNA tests currently available that are based on dominant or codominant mutations, including the $\mathrm{RHO}$ mutation for an autosomal dominant form of PRA in the English Mastiff offered by OptiGen (www.optigen.com) (Kijas et al. 2002), the mutation in HSF4 that is associated with hereditary cataract in the Australian Shepherd that is offered by the Animal Health Trust (www.AHT.org.uk) (Mellersh et al. 2006b, 2009), and the polymorphisms associated with renal dysplasia in multiple breeds that is offered by DoGenes (www.dogenes.com) (Whiteley et al. 2011).

\section{Complex conditions}

Genetically complex conditions that result from mutations in multiple genes or the interaction between genes and the environment represent a vastly different scenario from genetically simple traits. Individual polymorphisms will invariably be identified that increase an individual dog's risk of developing an associated condition but cannot predict with absolute certainty whether the dog will become clinically affected. Since the canine genome sequence became available, four different, increasingly dense, whole-genome SNP arrays have been developed [the $27 \mathrm{~K}(\mathrm{v} 1)$ and $50 \mathrm{~K}(\mathrm{v} 2)$ canine Affymetrix SNP chips and the Illumina CanineSNP20 and CanineHD BeadChips], providing increasingly sensitive means with which to identify regions of the genome associated with complex traits of interest. Several reports have been published that identify regions of the canine genome associated with complex traits (Bannasch et al. 2010; Wilbe et al. 2010), and precise variants underlying these associations will almost certainly be identified in the near future.
Associations between specific genes and complex conditions are obviously important and will make profound contributions toward our understanding of gene function and disease aetiology, but they present the scientist with a dilemma when it comes to deciding whether to make a DNA test available based on risk factors. There is a need for dog owners and breeders to balance the desire to breed for "genetic health" by eliminating disease-associated mutations from their breed with the need to maintain genetic diversity. As stated already, most dog breeders are not generally in a position to objectively evaluate the specific level of risk associated with a given mutation from a scientific publication. There will be a tendency for all mutations described as "risk factors" to be considered similarly, regardless of the level of risk they convey, and once a DNA test becomes commercially available, many breeders will have their dogs tested and avoid breeding with dogs that carry that mutation. It is, therefore, irresponsible of DNA test providers to offer tests for isolated mutations that are only weakly associated with disease; these mutations are likely to be minor modifiers of more major risk factors and eliminating them may reduce genetic diversity without dramatically reducing the prevalence of disease. Increasingly, research to identify canine disease-associated mutations is being funded by dog-oriented organisations, and researchers need to resist pressure to make DNA tests available prematurely to compensate these stakeholders for their financial and moral support.

\section{Summary}

Genetic tools available with which to dissect the canine genome are becoming increasingly sophisticated, and the number of disease-associated genetic variants that will be identified over the next few years will undoubtedly increase dramatically. These mutations will form the basis of extremely valuable tools that dog owners and breeders will clamour to use to eliminate both Mendelian and complex inherited conditions from their beloved breeds. If the public is to maintain confidence in these new tools, scientists must be responsible custodians of their findings, and make DNA tests commercially available only if they can be accompanied by straightforward, transparent, userfriendly advice on how they should be used to reduce the frequency of disease without reducing genetic diversity unnecessarily.

Open Access This article is distributed under the terms of the Creative Commons Attribution Noncommercial License which permits any noncommercial use, distribution, and reproduction in any medium, provided the original author(s) and source are credited. 


\section{Appendix}

\begin{tabular}{|c|c|}
\hline es & \\
\hline AHT & www.aht.org.uk (UK) \\
\hline $\begin{array}{l}\text { Alfort School of Veterinary } \\
\text { Medicine }\end{array}$ & www.labradorcnm.com (France) \\
\hline Antagene & www.antagene.com (France) \\
\hline Auburn University & www.vetmed.auburn.edu (USA) \\
\hline Cornell University & www.web.vet.cornell.edu/ (USA) \\
\hline Finnzymes & www.finnzymes.fi (Finland) \\
\hline Genindexe & www.genindexe.com (France) \\
\hline Genetic Technologies Ltd & $\begin{array}{l}\text { www.geneticscienceservices.com } \\
\text { (Australia) }\end{array}$ \\
\hline GenMARK & www.genmarkag.com (USA) \\
\hline Genoscoper & $\begin{array}{l}\text { http://www.genoscoper.com/ } \\
\text { in_english2/gene_tests/ (Finland) }\end{array}$ \\
\hline HealthGene & www.healthgene.com (Canada) \\
\hline Laboklin & www.laboklin.co.uk (UK) \\
\hline OFA & www.offa.org (USA) \\
\hline OptiGen & www.optigen.com (USA) \\
\hline PennGen & www.vet.upenn.edu (USA) \\
\hline University of California & $\begin{array}{l}\text { www.vgl.ucdavis.edu/services/ } \\
\text { index.php (USA) }\end{array}$ \\
\hline University of Missouri & $\begin{array}{l}\text { www.caninegeneticdiseases.net } \\
\text { (USA) }\end{array}$ \\
\hline University of Utrecht & $\begin{array}{l}\text { email: P.A.J.Leegwater@vet.uu.nl } \\
\text { (Holland) }\end{array}$ \\
\hline Van Haeringen & $\begin{array}{l}\text { email: info@vhlgenetics.com } \\
\text { (Holland) }\end{array}$ \\
\hline VDA & www.vetdnacenter.com (USA) \\
\hline VetGen & www.vetgen.com (USA) \\
\hline
\end{tabular}

\section{References}

Acland GM, Ray K, Mellersh CS, Gu W, Langston AA, Rine J, Ostrander EA, Aguirre GD (1998) Linkage analysis and comparative mapping of canine progressive rod-cone degeneration (prcd) establishes potential locus homology with retinitis pigmentosa (RP17) in humans. Proc Natl Acad Sci USA 96:3048-3053

Aguirre GD, Acland GM (1988) Variation in retinal degeneration phenotype inherited at the prcd locus. Exp Eye Res 46:663-687

Aguirre GD, Acland GM (2006) Models, mutants and man: searching for unique phenotypes and genes in the dog model of inherited retinal degeneration. In: Ostrander EA, Giger U, Lindblad-Toh K (eds) The dog and its genome. Cold Spring Harbor Laboratory Press, Cold Spring Harbor, pp 291-325

Awano T, Johnson GS, Wade CM, Katz ML, Johnson GC, Taylor JF, Perloski M, Biagi T, Baranowska I, Long S, March PA, Olby NJ, Shelton GD, Khan S, O'Brien DP, Lindblad-Toh K, Coates JR (2009) Genome-wide association analysis reveals a SOD1 mutation in canine degenerative myelopathy that resembles amyotrophic lateral sclerosis. Proc Natl Acad Sci USA 106:2794-2799
Bannasch D, Safra N, Young A, Karmi N, Schaible RS, Ling GV (2008) Mutations in the SLC2A9 gene cause hyperuricosuria and hyperuricemia in the dog. PLoS Genet 4:e1000246

Bannasch D, Young A, Myers J, Truve K, Dickinson P, Gregg J, Davis R, Bongcam-Rudloff E, Webster MT, Lindblad-Toh K, Pedersen N (2010) Localization of canine brachycephaly using an across breed mapping approach. PLoS ONE 5:e9632

Booij JC, Florijn RJ, ten Brink JB, Loves W, Meire F, van Schooneveld MJ, de Jong PT, Bergen AA (2005) Identification of mutations in the AIPL1, CRB1, GUCY2D, RPE65, and RPGRIP1 genes in patients with juvenile retinitis pigmentosa. J Med Genet 42:e67

Busse C, Barnett KC, Mellersh CS, Adams VJ (2011) Ophthalmic and cone derived electrodiagnostic findings in outbred miniature long-haired dachshunds homozygous for a RPGRIP1 mutation. Vet Ophthalmol 14:146-152

Curtis R (1983) Aetiopathological aspects of inherited lens dislocation in the Tibetan Terrier. J Comp Pathol 93:151-163

Curtis R (1990) Lens luxation in the dog and cat. Vet Clin North Am Small Anim Pract 20:755-773

Curtis R, Barnett KC (1980) Primary lens luxation in the dog. J Small Anim Pract 21:657-668

Curtis R, Barnett KC (1993) Progressive retinal atrophy in miniature longhaired dachshund dogs. Br Vet J 149:71-85

Curtis R, Barnett KC, Lewis SJ (1983) Clinical and pathological observations concerning the aetiology of primary lens luxation in the dog. Vet Rec 112:238-246

Dryja TP, Adams SM, Grimsby JL, McGee TL, Hong DH, Li T, Andreasson S, Berson EL (2001) Null RPGRIP1 alleles in patients with Leber congenital amaurosis. Am J Hum Genet 68:1295-1298

Durbin RM, Abecasis GR, Altshuler DL, Auton A, Brooks LD, Gibbs RA, Hurles ME, McVean GA (2010) A map of human genome variation from population-scale sequencing. Nature 467:10611073

Farias FH, Johnson GS, Taylor JF, Giuliano E, Katz ML, Sanders DN, Schnabel RD, McKay SD, Khan S, Gharahkhani P, O'Leary CA, Pettitt L, Forman OP, Boursnell M, McLaughlin B, Ahonen S, Lohi H, Hernandez-Merino E, Gould DJ, Sargan D, Mellersh CS (2010) An ADAMTS17 splice donor site mutation in dogs with primary lens luxation. Invest. Ophthalmol Vis Sci 51:4716-4721

Goldstein O, Zangerl B, Pearce-Kelling S, Sidjanin DJ, Kijas JW, Felix J, Acland GM, Aguirre GD (2006) Linkage disequilibrium mapping in domestic dog breeds narrows the progressive rodcone degeneration interval and identifies ancestral diseasetransmitting chromosome. Genomics 88:541-550

Gould D, Pettitt L, McLaughlin B, Holmes N, Forman O, Thomas A, Ahonen S, Lohi H, O'Leary C, Sargan D, Mellersh C (2011) ADAMTS17 mutation associated with primary lens luxation is widespread among breeds. Vet Ophthalmol 14:1-7

Gray H (1909) The diseases of the eye in domesticated animals. Vet $\operatorname{Rec} 21: 678$

Gray H (1932) Some medical and surgical conditions in the dog and cat. Vet Rec 12:1-10

Hameed A, Abid A, Aziz A, Ismail M, Mehdi SQ, Khaliq S (2003) Evidence of RPGRIP1 gene mutations associated with recessive cone-rod dystrophy. J Med Genet 40:616-619

Karmi N, Brown EA, Hughes SS, McLaughlin B, Mellersh CS, Biourge V, Bannasch DL (2010) Estimated frequency of the canine hyperuricosuria mutation in different dog breeds. $\mathrm{J}$ Vet Intern Med 24:1337-1342

Kijas JW, Cideciyan AV, Aleman TS, Pianta MJ, Pearce-Kelling SE, Miller BJ, Jacobson SG, Aguirre GD, Acland GM (2002) Naturally occurring rhodopsin mutation in the dog causes retinal dysfunction and degeneration mimicking human dominant retinitis pigmentosa. Proc Natl Acad Sci USA 99:6328-6333 
Mellersh CS, Boursnell ME, Pettitt L, Ryder EJ, Holmes NG, Grafham D, Forman OP, Sampson J, Barnett KC, Blanton S, Binns MM, Vaudin M (2006a) Canine RPGRIP1 mutation establishes cone-rod dystrophy in miniature longhaired dachshunds as a homologue of human Leber congenital amaurosis. Genomics 88:293-301

Mellersh CS, Pettitt L, Forman OP, Vaudin M, Barnett KC (2006b) Identification of mutations in HSF4 in dogs of three different breeds with hereditary cataracts. Vet Ophthalmol 9:369-378

Mellersh CS, McLaughlin B, Ahonen S, Pettitt L, Lohi H, Barnett KC (2009) Mutation in HSF4 is associated with hereditary cataract in the Australian Shepherd. Vet Ophthalmol 12:372-378

Miyadera K, Kato K, Aguirre-Hernandez J, Tokuriki T, Morimoto K, Busse C, Barnett K, Holmes N, Ogawa H, Sasaki N, Mellersh CS, Sargan DR (2009) Phenotypic variation and genotypephenotype discordance in canine cone-rod dystrophy with an RPGRIP1 mutation. Mol Vis 15:2287-2305

Morris RA, Dubielzig RR (2005) Light-microscopy evaluation of zonular fiber morphology in dogs with glaucoma: secondary to lens displacement. Vet Ophthalmol 8:81-84

Priat C, Hitte C, Vignaux F, Renier C, Jiang Z, Jouquand S, Cheron A, Andre C, Galibert F (1998) A whole-genome radiation hybrid map of the dog genome. Genomics 54:361-378

Turney C, Chong NH, Alexander RA, Hogg CR, Fleming L, Flack D, Barnett KC, Bird AC, Holder GE, Luthert PJ (2007) Pathological and electrophysiological features of a canine cone-rod dystrophy in the miniature longhaired dachshund. Invest Ophthalmol Vis Sci 48:4240-4249

Whiteley MH, Bell JS, Rothman DA (2011) Novel allelic variants in the canine cyclooxgenase-2 (Cox-2) promoter are associated with renal dysplasia in dogs. PLoS ONE 6:e16684

Wilbe M, Jokinen P, Truve K, Seppala EH, Karlsson EK, Biagi T, Hughes A, Bannasch D, Andersson G, Hansson-Hamlin H, Lohi H, Lindblad-Toh K (2010) Genome-wide association mapping identifies multiple loci for a canine SLE-related disease complex. Nat Genet 42:250-254

Willis MB, Curtis R, Barnett KC, Tempest WM (1979) Genetic aspects of lens luxation in the Tibetan terrier. Vet Rec 104: 409-412

Zangerl B, Goldstein O, Philp AR, Lindauer SJ, Pearce-Kelling SE, Mullins RF, Graphodatsky AS, Ripoll D, Felix JS, Stone EM, Acland GM, Aguirre GD (2006) Identical mutation in a novel retinal gene causes progressive rod-cone degeneration in dogs and retinitis pigmentosa in humans. Genomics 88:551-563

Zhao Y, Hong DH, Pawlyk B, Yue G, Adamian M, Grynberg M, Godzik A, Li T (2003) The retinitis pigmentosa GTPase regulator (RPGR)-interacting protein: subserving RPGR function and participating in disk morphogenesis. Proc Natl Acad Sci USA 100:3965-3970 